\title{
Use proper indicators of change in neuropsychological studies
}

\begin{abstract}
Some indicators have been proposed in biomedical research to index the reliable change of measurements after the intervention or treatment. A good indicator should have simple statistical property and can be easily used by people without formal statistical training. Our analysis shows that many mistakes occurred in medical publications in the application and interpretation of the indicators, especially in the statistical distributions of the indicators. In this paper we summarize some typical mistakes and give correct formulas.
\end{abstract}

Keywords: practice effect, normal distribution, linear regression
Volume 3 Issue 5 - 2017
Changyong Feng, ${ }^{1,2}$ Chongshu Chen,' Julia Z Zheng, ${ }^{3}$ Xiang Lu,' Bokai Wang,' Daryl I Smith, ${ }^{2}$ Hongyue Wang'

'Department of Biostatistics \& Computational Biology, University of Rochester Medical Center, USA ${ }^{2}$ Department of Anaesthesiology, University of Rochester Medical Center, USA

${ }^{3}$ Department of Immunology and Microbiology, McGill University, Canada

Correspondence: Changyong Feng, Department of Biostatistics \& Computational Biology, University of Rochester Medical Center, 601 Elmwood Ave., Rochester, NY 14642, USA, Tel 585-275-4263, Email feng@urmc.rochester.edu

Received: June 29, 2017| Published: July 27, 2017
Abbreviations: SDI, standard deviation index; RCI, reliable change index; SRB, standard regression-based; PE, practice effect

\section{Introduction}

Repeated measurements have been widely used to study a change of outcomes in clinical neuropsychology, see Chelune et al., ${ }^{1} \mathrm{Du}$ et al., ${ }^{2}$ Frerichs \& Tuokko, ${ }^{3}$ Hinton-Bayre, ${ }^{4}$ Jacobson \& Truax, ${ }^{5}$ Levine et al., ${ }^{6}$ Ouimet et al., ${ }^{7}$ Sherman et al. ${ }^{8}$ and Zahodne et al.. ${ }^{9}$ To measure the change at the individual level, we first need to define a meaningful indicator of the change. A well-defined indicator should be interpretable both biologically and statistically. The statistical distribution of the indicator should be easily accessible to biomedical investigators.

Recently, Duff ${ }^{10}$ reviewed some indicators that have been used in medical journals. After reading Duff 's review paper, we have found several serious mistakes. Some of the mistakes are associated with errors that have appeared in the reference papers cited by Duff. ${ }^{10}$ Our survey shows that the statistical distributions of the indicators discussed in Duff ${ }^{10}$ are wrong. These mistakes may lead to invalid inferences and cause serious problems for biomedical investigators without appropriate statistical background. The purpose of our paper is to point out some of these mistakes, to explain the reason of these mistakes, and further to correct these errors.

\section{Fundamentals of change indicators}

In order to have an appropriate definition of the indicator for the change, we first discuss some fundamental facts. For a randomly selected individual in the experimental group, let $T_{1}$ and $T_{2}$ denote the measurements at time 1 and time 2 , respectively. $T_{2}-T_{1}$ is the change from time 1 to time 2 . Let $\mathrm{M}_{1}$ and $\mathrm{M}_{2}$ be the population mean values of the measurements of the control group at times 1 and 2, respectively. The change of the individual effect in the experimental group after adjusting the practice effect $(\mathrm{PE})$ is $\left(\mathrm{T}_{2}-\mathrm{M}_{2}\right)-\left(\mathrm{T}_{1}-\mathrm{M}_{1}\right)=\left(\mathrm{T}_{2}-\right.$ $\left.\mathrm{T}_{1}\right)-\left(\mathrm{M}_{2}-\mathrm{M}_{1}\right)$, see Duff. ${ }^{10}$ Suppose the standard deviations of $\mathrm{T}_{1}$ and $\mathrm{T}_{2}$ are $\mathrm{S}_{1}$ and $\mathrm{S}_{2}$ with the correlation coefficient $\mathrm{r}_{12}$. Then the standard deviations of $\mathrm{T}_{2}-\mathrm{T}_{1}$ and $\left(\mathrm{T}_{2}-\mathrm{T}_{1}\right)-\left(\mathrm{M}_{2}-\mathrm{M}_{1}\right)$ are the same and they are equal to $\sqrt{S_{1}^{2}+S_{2}{ }^{2}-2 r_{12} S_{1} S_{2}}$. The standardized change and the standardized change after adjusting PE are respectively. These two formulas are the fundamentals of construction of indicators of change

$$
\begin{aligned}
& \frac{T_{2}-T_{1}}{\sqrt{S_{1}^{2}+S_{2}^{2}-2 r_{12} S_{1} S_{2}}} \\
& \frac{\left(T_{2}-T_{1}\right)-\left(M_{2}-M_{1}\right)}{\sqrt{S_{1}^{2}+S_{2}^{2}-2 r_{12} S_{1} S_{2}}}
\end{aligned}
$$

\section{Mistakes in the use of indicators in medical publications}

In this paper, we focus on two types of mistakes that have appeared in Duff: ${ }^{10}$

a. Order of operations

b. Statistical distribution of the indicators. Similar mistakes also appeared in some publications cited in Duff. ${ }^{10}$

\section{Order of operations}

In Equation 1 of Duff, ${ }^{10}$ the formula of the standard deviation index (SDI) is defined as

$$
S D I=T_{2}-T_{1} / \mathrm{S}_{1}
$$


Order of operations is a fundamental part of elementary school math, see for example Ewen \& Nelson. ${ }^{11}$ One of the rules of the order of operations is that multiplication and division have higher priority than addition and subtraction. According to this rule, the right hand side of (1) is the same as

$$
T_{2}-\left(T_{1} / \mathrm{S}_{1}\right)
$$

For example 80-94.5/11.46 $=80-(94.5 / 11.46)=80-8.25=71.75$.

Anybody with knowledge of elementary school math should obtain this result. However, on p. 255 of Duff, ${ }^{10}$ the calculation is 80 $94.5 / 11.46=-1.26$, which is totally wrong.

A closer look at the Duff ${ }^{10}$ 's result found that to calculate 80 $94.5 / 11.46$, he first calculated $80-94-5$, which is -14.5 , and then divided it by 11.46 , which is -1.27 . The rules of the order of operations are not fulfilled here and it appears that the incorrect assumption made that $\mathrm{T}_{2}-\mathrm{T}_{1} / \mathrm{S}_{1}$ is the same as $\left(\mathrm{T}_{2}-\mathrm{T}_{1}\right) / \mathrm{S}_{1}$.

It is clear that the author does not understand the rules of the order of operations. He simply assumes that $\mathrm{T}_{2}-\mathrm{T}_{1} / \mathrm{S}_{1}$ is the same as $\left(\mathrm{T}_{2}-\mathrm{T}_{1}\right) /$ $\mathrm{S}_{1}$. In fact, for the measurement data, the right hand side of (1) appears to be in error. For example, suppose the unit of $T_{1}$ and $T_{2}$ is kilometer $(\mathrm{km})$. Then the unit of $\mathrm{S}_{1}$ is also $\mathrm{km}$. Physically, then the expression $\mathrm{T}_{2}-\left(\mathrm{T}_{1} / \mathrm{S}_{1}\right)$ is meaningless.

In the following discussion we examine Duff ${ }^{10}$ 's formulas with the appropriate orders of operations.

For example, the SDI should be of the form

$$
S D I=\left(T_{2}-T_{1}\right) / \mathrm{S}_{1}
$$

Equation 1 of Duff ${ }^{10}$ also reported several other indicators, which we assume are of the following forms:

a. The reliable change index (RCI)

$$
R C I=\left(T_{2}-T_{1}\right) / S E D
$$

b. The RCI controlling for practice effect (PE)

$$
R C I P E=\left[\left(T_{2}-T_{1}\right)-\left(\mathrm{M}_{2}-M_{1}\right)\right] / \mathrm{SED}
$$

c. The Iverson ${ }^{11} \mathrm{RCI}$ controlling for practice effect $(\mathrm{PE})$ :

$$
R C I^{\prime}{ }_{P E}=\left[\left(T_{2}-T_{1}\right)-\left(M_{2} M_{1}\right)\right] / S E D_{\text {Iverson }} ;^{11}
$$

d. The standard regression-based (SRB) change formula

$$
S R B=\left(T_{2}-T_{1}\right) / \mathrm{SEE}
$$

The expression of SED, SED Iverson, ${ }^{11}$ and SEE will be discussed in relevant sections

\section{Statistical distributions of indicators}

The paragraph designated 'Methods for Assessing Reliable Change' on p.253 in Duff, ${ }^{10} \mathrm{~T}_{1}$ and $\mathrm{T}_{2}$ are said to be the scores at times 1 and 2, respectively; and $\mathrm{S}_{1}$ and $\mathrm{S}_{2}$ the standard deviations of control group at time 1 and time 2, respectively. Under Equation 1 on the same page, $\mathrm{S}_{1}$ and $\mathrm{S}_{2}$ are said to be the standard deviations at time 1 and time 2. According to the paper, there is one control group and one experimental group. It seems that $T_{1}$ and $T_{2}$ should be measurements at times 1 and 2 of the experimental group, as the outcome of interest is the change of the experimental group. If this is true, $\mathrm{S}_{1}$ and $\mathrm{S}_{2}$ should be the standard deviations of the experimental group instead of the control group at times 1 and 2, respectively. It is not reasonable to standardize the measurement of the experimental group using the standard deviation of the control group unless we assume these two groups have the same standard deviations.

As discussed in sections above, the standard deviation of $T_{2}-T_{1}$ is $\sqrt{S_{1}^{2}+S_{2}^{2}-2 r_{12} S_{1} S_{2}}$. If $\left(\mathrm{T}_{1}, \mathrm{~T}_{2}\right)$ has a bivariate normal distribution, then $\mathrm{T}_{2}-\mathrm{T}_{1}$ also follows a normal distribution. If we further assume that there is no change between times 1 and 2 (which means $T_{1}$ and $T_{2}$ have the same mean values), then has the standard normal distribution.

$$
\frac{T_{2}-T_{1}}{\sqrt{S_{1}^{2}+S_{2}^{2}-2 r_{12} S_{1} S_{2}}}
$$

\section{We discuss the distributions of some indicators listed in Equation I in Duff. IO}

The distribution of SDI in formula (3): From above we know that SDI in (3) does not follow standard normal distribution. We cannot compare the SDI with the quantile of the standard normal distribution to see whether there is a significant change from time 1 to time 2 . Unfortunately, on p.254, Duff ${ }^{10}$ assumes SDI has the standard normal distribution, which appears to be in error.

The distribution of RCI in formula (4): In Equation 1 of Duff, ${ }^{10}$ Hence, RCI does not follow the standard normal distribution. It also does not make sense to compare it with the quantile of the standard normal distribution to check if there is signi cant change from time 1 to time 2 .

The distribution of the Iverson RCI in formula (6): According to Equation 1 in Duff, ${ }^{10}$ Hence, RCI'PE does not follow the standard normal distribution either. We cannot compare RCI'PE statistic with the quantile of the standard normal distribution to evaluate if there is a significant change from time 1 to time 2 .

The distribution of SRB in formula (6): It is trivial to prove that $\mathrm{b}_{\text {est }}=\mathrm{r}_{12} \mathrm{~S}_{2} / \mathrm{S}_{1}$, not $\mathrm{S}_{2} / \mathrm{S}_{1}$ as has been reported in Equation 1 in Duff, ${ }^{10}$ and $S E E_{\text {est }}=S_{2} \sqrt{1+r_{12}^{2}}$. The formula of $\mathrm{SEE}_{\text {est }}$ in Equation 1 of Duff ${ }^{10}$ appears to be incorrect.

Our discussions indicate that none of the indicators reviewed in Equation 1 of Duff ${ }^{10}$ follow standard normal distribution. Unfortunately, Duff $^{10}$ and some medical publications compared those indicators with the quantile of the standard normal distribution to evaluate the change from time 1 to time 2 . This practice is inappropriate and should be avoided in future medical research.

\section{Correct formulas of indicators}

In this section we present correct formulations for these indicators listed in Duff. ${ }^{10}$ With these formulas, the investigators can compare statistics to the appropriate quantiles of the standard normal distribution to check whether a reliable change has occurred between time 1 and time 2. Note that all indicators discussed above are in the form of the critical part is to find the correct standard deviation of the change.

$\frac{\text { change from time } 1 \text { to time } 2}{\text { standard deviation of the change }}$




\section{Here are correct forms of those indicators}

The standard deviation index (SDI)

$$
S D I=\frac{T_{2}-T_{1}}{\sqrt{S_{1}^{2}+S_{2}^{2}-2 r_{12} S_{1} S_{2}}}
$$

The RCI controlling for practice e ect (PE)

$$
R C I_{P E}=\frac{\left(T_{2}-T_{1}\right)-\left(\mathrm{M}_{2}-M_{1}\right)}{\sqrt{S_{1}^{2}+S_{2}^{2}-2 r_{12} S_{1} S_{2}}}
$$

a. The standard regression-based (SRB) change

$$
S R B=\frac{\left(T_{2}-M_{2}\right)-\left(\mathrm{r}_{12} S_{2} / \mathrm{S}_{1}\right)\left(\mathrm{T}_{1}-M_{1}\right)}{S_{2} \sqrt{1-r_{12}^{2}}}
$$

Since the numerators of the RCI and the Iverson ${ }^{11} \mathrm{RCI}$ are exactly the same as those of SDI and RCIPE, respectively, we no longer need to introduce them.

\section{Conclusion}

In this paper, we point out some common mistakes related to indicators of change used in the medical literature. Some authors utilize (active language) the wrong distribution to compare the indicators and to determine whether a reliable change has occurred. Some indicators were redundant and not well defined.

\section{Acknowledgements}

None.

\section{Conflict of interest}

The author declares no conflict of interest.

\section{References}

1. Chelune GJ, Naugle RI, Luders H, et al. Individual change after epilepsy surgery: Practice effects and base-rate information. Neuropsychology. 1993;7(1):41-52.
2. Duff K, Beglinger LJ, Schultz SK, et al. Practice effects in the prediction of long-term cognitive outcome in three patient samples: A novel prognostic index. Arch Clin Neuropsychol. 2007;22(1):15-24.

3. Frerichs RJ, Tuokko HA. A comparison of methods for measuring cognitive change in older adults. Arch Clin Neuropsychol. 2005;20(3):321333.

4. Hinton-Bayré AD. Methodology is more important than statistics when determining reliable change. J Int Neuropsychol Soc. 2005;11(6):788789.

5. Jacobson NS, Truax P. Clinical significance: A statistical approach to defining meaningful change in psychotherapy research. J Consult Clin Psychol. 1991;59(1):12-19.

6. Levine AJ, Hinkin $\mathrm{CH}$, Miller EN, et al. The generalizability of neurocognitive test/retest data derived from a nonclinical sample for detecting change among two HIV+ cohorts. J Clin Exp Neuropsychol. 2007;29(6):669-678.

7. Ouimet LA, Stewart A, Collins B, et al. Measuring neuropsychological change following breast cancer treatment: An analysis of statistical models. J Clin Exp Neuropsychol. 2009;31(1):73-89.

8. Sherman EM, Wiebe S, Fay-McClymont TB, et al. Neuropsychological outcomes after epilepsy surgery: Systematic review and pooled estimates. Epilepsia. 2011;52(5):857-869.

9. Zahodne LB, Okun MS, Foote KD, et al. Cognitive declines one year after unilateral deep brain stimulation surgery in Parkinsons disease: A controlled study using reliable change. Clin Neuropsychol. 2009;23(3):385-405.

10. Duff K. Evidence-based indicators of neuropsychological change in the individual patient: relevant concepts and methods. Arch Clin Neuropsychol. 2012;27(3):248-261.

11. Ewen D, Nelson CR. Elementary Technical Mathematics. 11th ed. USA: Belmont; 2007.

12. Iverson GL. Interpreting change on the WAIS-III/WMS-III in clinical samples. Arch Clin Neuropsychol. 2001;16(2):183-191. 